\title{
A VARIABLE-LENGTH DHT-BASED FFT/IFFT PROCESSOR FOR VDSL/ADSL SYSTEMS
}

\author{
Tsung-Chieh Pao*, Ching-Chi Chang, and Chorng-Kuang Wang \\ Graduate Institute of Electronics Engineering and Department of Electrical Engineering, \\ National Taiwan University, Taipei 106, Taiwan R.O.C.
}

\begin{abstract}
In this paper, a matrix form of the radix-4 Discrete Hartly Transform (DHT) algorithm is derived. And two Process Element (PE) circuits are proposed for the variable-length application. One is for radix-2 with one real multiplier and three adders, and the other is for radix- 4 with one real multiplier and seven adders. The radix-4 DHT processor of variable-length, namely, $8192 / 4096 / 2048 / 1024 / 512$, meets the DMT-VDSL and ADSL requirements. Only six real multipliers, $2.01 \mathrm{~N}$ (16467) registers and simple logic circuits are used to perform 8192-points FFT/IFFT in $231 \mu \mathrm{s}$. Implemented in $0.25 \mu \mathrm{m} 1 \mathrm{P} 5 \mathrm{M}$ typical CMOS technology, the core occupies an active area of $5 \mathrm{~mm} \times 5 \mathrm{~mm}$. Power dissipation is $300 \mathrm{~mW}$ using $2.5 \mathrm{~V}$ supply voltage.
\end{abstract}

\section{INTRODUCTION}

Among standards with Multiple Carrier Modulation (MCM) technology using FFT/IFFT as the modulation technique, Very-high-bit-rate Digital Subscriber Line (VDSL) has potential for broadband communication of the next generation, owing to the popularity of twisted pairs [1]. The Discrete Fourier Transform (DFT) plays an important role in DMT/OFDM systems. Because of the computational complexity of $N$-points DFT is $\mathrm{O}\left(N^{2}\right)$, it takes large operation time and power consumption to perform DFT directly, especially in large transform size (e.g. 8192 points in VDSL). Hence, various FFT algorithms have been proposed to reduce the computational complexity [2], such as Cooley-Turkey algorithm, Winograd Algorithm (WFTA), radar algorithm and Prime Factor Algorithm (PFA). Cooley-Turkey algorithm is the most popular one in VLSI implementation due to reducing the computational complexity from $\mathrm{O}\left(N^{2}\right)$ to $\mathrm{O}\left(N \log _{r} N\right)$ and its regularity. Table 1 summaries several FFT architectures including Single-Path Delay Feedback (SDF) architecture [3], Multiple-Path Delay Commutator (MDC) [4], and DHT-based FFT/IFFT processor [5]. There are two key points shown in this table. One is that MDC architectures need more hardware than SDF architectures obviously. The other is that no matter what radix- $r$ SDF architecture is used, $2(N-1)$ registers are essential. In order to reduce hardware in FFT/IFFT processor, the design goal should be to reduce the number of real multipliers without increasing the number of registers. Based on the advantage of Hermitian symmetric property in DMT-VDSL and ADSL systems, some DHT-based FFT algorithms and architectures have been presented to reduce the numbers of real multipliers [5-7]. But there are some drawbacks about these architectures such as more buffers for RAM-based designs and only radix-2 DHT-based FFT algorithm is delivered. 'If the higher radix FFT algorithm is adopted, the fewer multipliers can be employed with Cooley-Turkey algorithm. Our goal is to derive matrix form of higher radix DHT algorithm from previous work and then implement it using less hardware.

The organization of this paper is as follows. The matrix form of radix-4 DIF DHT algorithm is derived in Section 2. Section 3 presents the proposed circuit designs of the two PEs. Section 4 shows VLSI implementation and performance summary of the FFT processor for VDSL system, and the conclusion is given in section 5 .

Table 1. Hardware comparisons of various FFT architectures.

\begin{tabular}{|c|c|c|c|}
\hline & $\begin{array}{c}\text { Real } \\
\text { Multipliers }\end{array}$ & Memory & $\begin{array}{c}\text { Through-put } \\
\text { (sample/cycle) }\end{array}$ \\
\hline Radix-2 SDF & $3\left(\log _{2} N-2\right)$ & $2(N-1)$ & 1 \\
\hline Radix-4 SDF & $3\left(\log _{4} N-1\right)$ & $2(N-1)$ & 1 \\
\hline Radix-8 SDF & $3\left(\log _{8} N-1\right)$ & $2(N-1)$ & 1 \\
\hline Radix-2 MDC & $3\left(\log _{2} N-2\right)$ & $2(1.5 N-2)$ & 2 \\
\hline Radix-4 MDC & $3\left(3 \log _{4} N-3\right)$ & $2(2.5 N-4)$ & 4 \\
\hline Radix-8 MDC & $3\left(7 \log _{8} N-7\right)$ & $2(4.5 N-8)$ & 8 \\
\hline $\begin{array}{c}\text { Radix-2 DHT- } \\
\text { based [5] }\end{array}$ & $\log _{2} N-1$ & $2(N-1)$ & 1 \\
\hline
\end{tabular}

\section{RADIX-4 DHT ALGORITHM}

Given $N$-points DHT definition as

$$
\begin{aligned}
y_{k} & =\sum_{n=0}^{N-1} x_{n}\left(\cos \frac{2 \pi k n}{N}+\sin \frac{2 \pi k n}{N}\right) \\
& =\sum_{n=0}^{N-1} x_{n} \operatorname{cas} \frac{2 \pi k n}{N},
\end{aligned}
$$

This program is supported by the National Science Council (NSC), Taiwan R.O.C..

*Currently with Realtek Semiconductor Corp. 
where $k=0,1, \ldots, N-1$. The frequency samples, $y_{4 k}, y_{4 k+1}$, $y_{4 k+2}$ and $y_{4 k+3}$ are derived as [8]:

$$
\begin{aligned}
y_{4 k} & =\sum_{n=0}^{N-1} x_{n} \operatorname{cas} \frac{2 \pi(4 k) n}{N} \\
= & \sum_{n=0}^{N / 4-1}\left[x_{n}+x_{n+\frac{N}{4}}+x_{n+\frac{N}{2}}+x_{n+\frac{3 N}{4}}\right] \operatorname{cas} \frac{2 \pi(4 k) n}{N}, \\
y_{4 k+1} & =\sum_{n=0}^{N-1} x_{n} \operatorname{cas} \frac{2 \pi(4 k+1) n}{N} \\
= & \sum_{n=0}^{N / 4-1}\left[\left(x_{n}-x_{n+\frac{N}{2}}+x_{\frac{N}{4}-n}-x_{\frac{3 N}{4}-n}\right) \cos \frac{2 \pi n}{N}+\right. \\
& \left.\left(x_{n+\frac{3 N}{4}}-x_{n+\frac{N}{4}}+x_{\frac{N}{2}-n}-x_{N-n}\right) \sin \frac{2 \pi n}{N}\right] \operatorname{cas} \frac{2 \pi(4 k) n}{N}, \\
y_{4 k+2}= & \sum_{n=0}^{N-1} x_{n} \operatorname{cas} \frac{2 \pi(4 k+2) n}{N} \\
= & \sum_{n=0}^{N / 4-1}\left[\left(x_{n}+x_{n+\frac{N}{2}}-x_{n+\frac{N}{4}}-x_{n+\frac{3 N}{4}}\right) \cos \frac{2 \pi 2 n}{N}+\right. \\
& \left.\left(x_{\frac{N}{4}-n}+x_{\frac{3 N}{4}-n}-x_{\frac{N}{2}-n}-x_{N-n}\right) \sin \frac{2 \pi 2 n}{N}\right] \operatorname{cas} \frac{2 \pi(4 k) n}{N}, \\
y_{4 k+3}= & \sum_{n=0}^{N-1} x_{n} \operatorname{cas} \frac{2 \pi(4 k+3) n}{N} \\
= & \sum_{n=0}^{N / 4-1}\left[\left(x_{n}-x_{n+\frac{N}{2}}-x_{\frac{N}{4}-n}+x_{\frac{3 N}{4}-n}\right) \cos \frac{2 \pi 3 n}{N}+\right. \\
& \left.\left(-x_{n+\frac{3 N}{4}}+x_{n+\frac{N}{4}}+x_{\frac{N}{2}-n}-x_{N-n}\right) \sin \frac{2 \pi 3 n}{N}\right] \operatorname{cas} \frac{2 \pi(4 k) n}{N},
\end{aligned}
$$

where $k=0,1, \ldots, N / 4-1$. From Eq. 2, 3, 4 and 5, the frequency samples, $y_{4 k}, y_{4 k+1}, y_{4 k+2}$ and $y_{4 k+3}$, are respectively equal to the DHT of the four N/4-points sequences bracketed in these four equations. Then, four N/4 $\times 1$ matrices, $\boldsymbol{Y}_{4 k}, \boldsymbol{Y}_{4 \mathrm{k}+1}, \boldsymbol{Y}_{4 k+2}$ and $\boldsymbol{Y}_{4 k+3}$, and four $N / 4 \times N$ matrices, $\boldsymbol{X}_{A}, \boldsymbol{X}_{B}, \boldsymbol{X}_{C}$ and $\boldsymbol{X}_{D}$, are defined as follows.

$\boldsymbol{Y}_{4 k}=\left[\begin{array}{lllll}y_{0} & y_{4} & y_{8} & \ldots & y_{4(N / 4-1)}\end{array}\right]^{\mathrm{T}}$,

$\boldsymbol{Y}_{4 k+1}=\left[\begin{array}{llllll}y_{1} & y_{5} & y_{9} & \ldots & y_{4(N / 4-1)+1}\end{array}\right]^{\mathrm{T}}$

$\boldsymbol{Y}_{4 k+2}=\left[\begin{array}{lllll}y_{2} & y_{6} & y_{10} & \ldots & y_{4(N / 4-1)+2}\end{array}\right]^{\mathrm{T}}$,

$Y_{4 k+3}=\left[\begin{array}{llllll}y_{3} & y_{7} & y_{11} & \ldots & y_{4(N / 4-1)+3}\end{array}\right]^{\mathrm{T}^{\prime}}$,

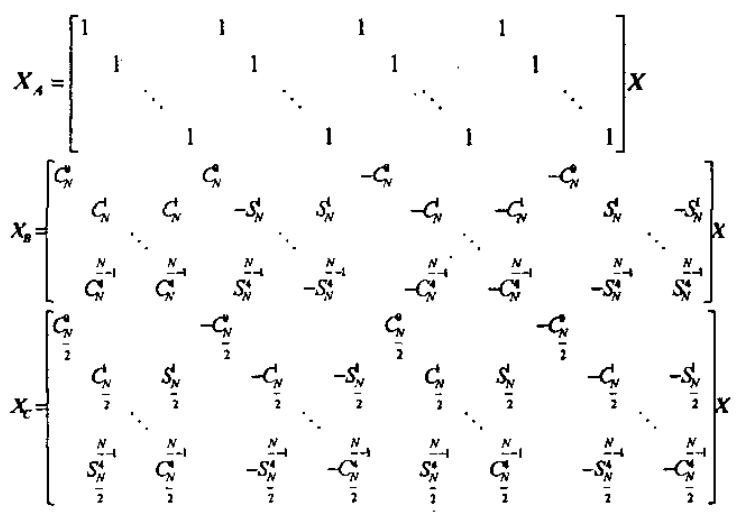

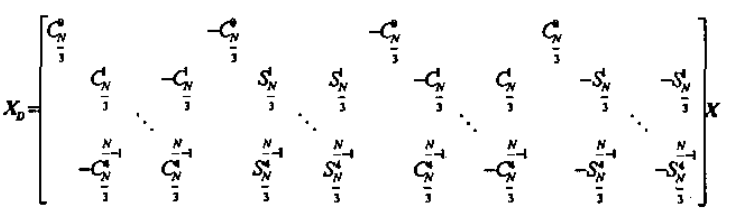

where $X=\left[\begin{array}{llllll}x_{0} & x_{1} & x_{2} & \ldots & x_{N-1}\end{array}\right]^{\mathrm{T}}$, and $C_{N}{ }^{k}=\cos (2 \pi k n / N)$ and $S_{N}{ }^{k}=\sin (2 \pi k n / N)$. The matrix form of DHT is

$$
\left[\begin{array}{c}
Y_{4 k} \\
Y_{4 k+1} \\
Y_{4 k+2} \\
Y_{4 k+3}
\end{array}\right]=\left[\begin{array}{cccc|c}
H(N / 4) & 0 & 0 & 0 \\
0 & H(N / 4) & 0 & 0 & X_{A} \\
X_{B} \\
0 & 0 & H(N / 4) & 0 & X_{C} \\
X_{D}
\end{array}\right]
$$

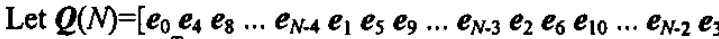
$\left.e_{7} e_{11} \ldots e_{N-1}\right]^{\mathrm{T}}$ be the permutation matrix, and $e_{i}$ denotes an $N \times 1$ column matrix whose $(i+1)$ th element is 1 . Then Eq. 6 can be derived as a similar form of Radix-2 DHT [6], as shown in the following.

$Q(N) Y=\left[\begin{array}{c}Y_{4 k} \\ Y_{4 k+1} \\ Y_{4 t+2} \\ Y_{t+3}\end{array}\right]=\left[\begin{array}{cccc}H(N / 4) & 0 & 0 & 0 \\ 0 & H(N / 4) & 0 & 0 \\ 0 & 0 & H(N / 4) & 0 \\ 0 & 0 & 0 & H(N / 4)\end{array}\right] P(N) X$,

$\boldsymbol{H}_{N / M}(M)$ is the direct sum of $N / M M \times M$ matrices, $\boldsymbol{H}(M)$.

$$
\begin{aligned}
\boldsymbol{H}_{N / M}(M) & =\boldsymbol{H}(M) \oplus \boldsymbol{H}(M) \oplus \cdots \oplus \boldsymbol{H}(M) \\
& =\left[\begin{array}{cccc}
\boldsymbol{H}(M) & 0 & \cdots & 0 \\
\boldsymbol{0} & \boldsymbol{H}(M) & \cdots & 0 \\
\vdots & \vdots & \ddots & \vdots \\
\boldsymbol{0} & 0 & \cdots & \boldsymbol{H}(M)
\end{array}\right]
\end{aligned}
$$

Eq. 7 can be simplified as

$$
\boldsymbol{Q}_{1}(N) \boldsymbol{Y}=\boldsymbol{H}_{4}(N / 4) \boldsymbol{P}_{1}(N) \boldsymbol{X},
$$

which represents that the $N$-points DHT of $\boldsymbol{X}$ becomes four parts of $N / 4$-points DHT of $\boldsymbol{P}_{1}(N) \boldsymbol{X}$. And the order of the DHT result, $\boldsymbol{Y}$, is permuted to $\boldsymbol{Q}_{1}(N) \boldsymbol{Y}$. Repeating the similar decomposition, radix -4 DHT can be gotten as

$$
Q_{N / 4}(4) Q_{N / 6}(16) \cdots Q_{I}(N) Y=P_{N / 4}(4) P_{N / 6}(16) \cdots P_{f}(N) X
$$

It shows that the $N$-points DHT of $\boldsymbol{X}$ can be performed by several multiplications of matrix, $\boldsymbol{P}_{N / M}(M)$, which is corresponding to the $\left(\log _{4} N / M+1\right)$ th stage of the DHT algorithm. And the order of DHT result is bit-reversed of $\boldsymbol{Y}$.

\section{CIRCUIT DESIGNS}

To realize the 8192-points FFT for VDSL, one radix-2 DHT stage and six radix-4 DHT stages are needed as shown in Fig. 1. Besides, an external dual-port RAM and two adders are required to perform the rearrangement of bit-reversed DHT results and acquire the normal-order FFT results [7]. 


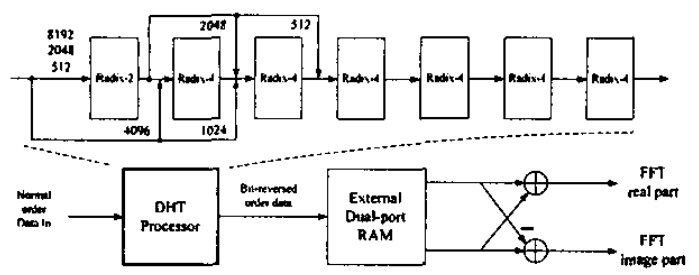

Fig. 1. Variable-length DHT-based FFT processor for 8192/4096/2048/1024/512-points VDSL and 512-points ADSL.

\subsection{Radix-2 PE}

Based on the matrix form of radix-2 DIF DHT algorithm [6],

$$
\boldsymbol{Z}=\boldsymbol{Q}_{N / 2}(2) \boldsymbol{Q}_{N / 4}(4) \cdots \boldsymbol{Q}_{1}(N) \boldsymbol{Y}=\boldsymbol{P}_{N / 2}(2) \boldsymbol{P}_{N / 4}(4) \cdots \boldsymbol{P}_{l}(N) X,
$$

the $\boldsymbol{X}$ column matrix denotes the sequential input data in time domain, and the $Z$ column matrix indicates the sequential output data in bit-reversed order. The matrix, $\boldsymbol{P}_{N / M}(M)$, is corresponding to the $\left(\log _{2} N / M+1\right)$ th stage $\mathrm{PE}$ And it has the general form as Eq. 12 [6]. Fig. 2 shows the proposed circuit architecture of radix-2 DIF DHT PE, $\boldsymbol{P}_{N M M}(M)$. Due to the utilization of the two-phase operating technique, only one real multiplier is employed to perform two multiplications.

$$
\begin{aligned}
& P(M)=\left[\begin{array}{cc}
I_{M / 2} & 0 \\
0 & K(M / 2)
\end{array}\right]\left[\begin{array}{cc}
I_{M / 2} & I_{M / 2} \\
I_{M / 2} & -I_{M / 2}
\end{array}\right] \\
& K(M / 2)=C(M / 2)+S(M / 2) \\
& =\left[\begin{array}{llll}
C_{M}^{n} & & & \\
& C_{M}^{1} & & \\
& & \ddots & \\
& & & C_{\mu}^{\mu / 2-1}
\end{array}\right]+\left[\begin{array}{llll}
S_{M}^{a} & & & \\
& & & S_{M}^{\prime} \\
& S_{\mu}^{\mu / 2-1} &
\end{array}\right]
\end{aligned}
$$

\subsection{Radix-4 PE}

The matrix form of radix-4 DIF DHT algorithm is followed as Eq. 10 and the matrix, $\boldsymbol{P}_{N M M}(M)$, is corresponding to the $\left(\log _{4} N / M+1\right)$ th stage PE. As the sequential input data, $\boldsymbol{X}$, enter each PE stage, the input data are rearranged by the circuit as shown in Fig. 3. The 16-points radix-4 DHT is demonstrated in the following.

$$
\begin{aligned}
Z=Q_{1}(4) Q_{1}(16) Y=P_{4}(4) P_{1}(16) X, \text { where } \\
P_{I}(16)=\left[\begin{array}{cccc}
I & I & I & I \\
B & C & -B & -C \\
A & -A & A & -A \\
D & E & -D & -E
\end{array}\right] \\
I=\left[\begin{array}{cccc}
1 & 0 & 0 & 0 \\
0 & 1 & 0 & 0 \\
0 & 0 & 1 & 0 \\
0 & 0 & 0 & 1
\end{array}\right], \quad A=\left[\begin{array}{cccc}
C_{16 / 2}^{0} & 0 & 0 & 0 \\
0 & C_{16 / 2}^{1} & 0 & S_{16 / 2}^{2} \\
0 & 0 & C_{16 / 2}^{2}+S_{16 / 2}^{2} & 0 \\
0 & S_{16 / 2}^{3} & 0 & C_{16 / 2}^{3}
\end{array}\right] \\
B=\left[\begin{array}{ccccc}
C_{16 / 1}^{0} & 0 & 0 & 0 \\
0 & C_{16 / 1}^{1} & 0 & C_{16 / 1}^{1} \\
0 & 0 & C_{16 / 1}^{2}+C_{16 / 1}^{2} & 0 \\
0 & C_{16 / 1}^{3} & 0 & C_{16 / 1}^{3}
\end{array}\right], C=\left[\begin{array}{cccc}
C_{36 / 1}^{0} & 0 & 0 & 0 \\
0 & -S_{16 / 1}^{1} & 0 & S_{16 / 1}^{1} \\
0 & 0 & S_{16 / 1}^{2}-S_{16 / 1}^{2} & 0 \\
0 & S_{16 / 1}^{3} & 0 & -S_{16 / 1}^{3}
\end{array}\right]
\end{aligned}
$$

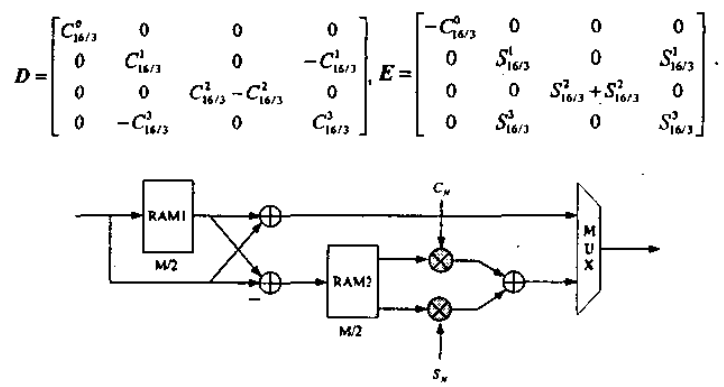

Fig. 2. Radix-2 DIF DHT PE.

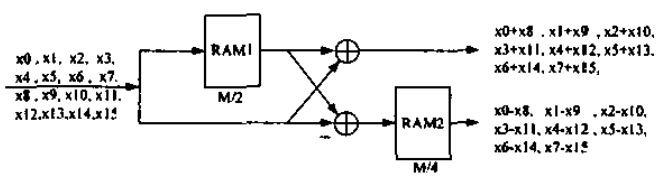

Fig. 3. Rearrangement of each stage input (e.g. $M=16$, for $\boldsymbol{P}_{1}(16)$ ).

After the rearrangement circuit, the $P E, P_{1}(16) X$, becomes two simplified matrices shown as Eq. 14 and 15.

$$
\begin{aligned}
& P_{1}(16) X=\left[\begin{array}{c}
a_{4 k} \\
a_{4 k+1} \\
a_{4 k+2} \\
a_{4 k+3}
\end{array}\right] \text {, where } \\
& {\left[\begin{array}{c}
\boldsymbol{a}_{\langle k} \\
\boldsymbol{a}_{4 k+2}
\end{array}\right]=\left[\begin{array}{cc}
\boldsymbol{I} & \boldsymbol{I} \\
\boldsymbol{A} & -\boldsymbol{A}
\end{array}\right]\left[\begin{array}{llll}
x_{0}+x_{8} & x_{1}+x_{9} & \cdots & x_{7}+x_{15}
\end{array}\right]^{T}} \\
& {\left[\begin{array}{l}
\boldsymbol{a}_{4 k+1} \\
\boldsymbol{a}_{4 k+3}
\end{array}\right]=\left[\begin{array}{cc}
\boldsymbol{B} & \boldsymbol{C} \\
\boldsymbol{D} & \boldsymbol{E}
\end{array}\right]\left[\begin{array}{llll}
x_{0}-x_{8} & x_{1}-x_{9} & \cdots & x_{7}-x_{15}{ }^{\Gamma}
\end{array}\right.}
\end{aligned}
$$

In Eq. 14, this part of $\boldsymbol{P}_{1}(16) \boldsymbol{X}$ is the same as the general form of radix-2 DIF DHT PE derived in Eq. 12. The similar circuit architecture shown in Fig. 2 can be utilized. In Eq. 15, this part of $\boldsymbol{P}_{1}(16) X$ is performed by the circuit architecture as shown in Fig. 4. The two-phase multiplication of single multiplier technique is also employed.

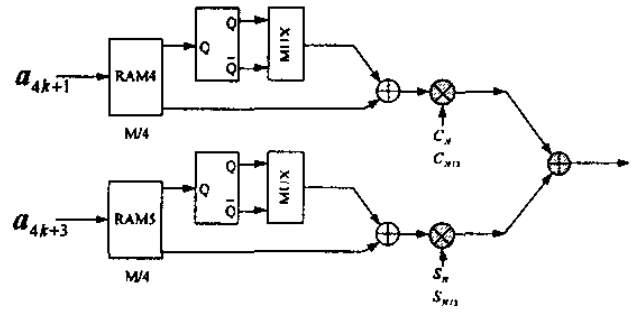

Fig. 4. Circuit architecture for computing $a_{4 k+1}$ and $a_{4 k+3}$.

The circuits in Fig. 2 and 4 for radix-4 DHT use the same multiplier because of performing the multiplication at different cycles. By rearranging the computing order as $a_{4 k}, a_{4 k+2}, a_{4 k+1}$ and $a_{4 k+3}$, the bit-reversed order in $Z$ can be obtained. 
As computing different points of radix-4 DHT, only the size of $M$ in Fig. 2 and 4 is changed. The radix-4 DHT PE can be performed with latency of $3 M / 4$ cycles by only one real multiplier, seven real adders, $3 M / 4$ RAM as delay line, $3 M / 4$ RAM with double clock rate, two ROMs as sine and cosine coefficients, and some simple logic circuits.

\section{VLSI IMPLEMENTATION AND PERFORMANCE SUMMARY}

For a variable-length DHT-based FFT processor, several considerations in VLSI implementation such as variable-length, simplified ROM of $\sin / \cos$ tables, cyclic extension removing and fixed-points evaluation for SNR requirements of VDSL system are all taken into account in this chip. The radix-4 DIF DHT processor is implemented with $0.25 \mu \mathrm{m} 1 \mathrm{P} 5 \mathrm{M}$ typical CMOS technology. Chip design and simulation summary are described in Table 2, and chip layout is shown in Fig. 5 . Compared in Table 1, it shows that the proposed radix -4 DHT-based FFT architecture has the fewest numbers of real multipliers among SDF architectures and radix-2 DHT-based FFT processor [5]. Otherwise, they use almost the same numbers of registers. Table 3 shows the comparisons with other 8192-points FFT chips.

Table 2. Chip design and simulation summary.

\begin{tabular}{|c|c|}
\hline Process & CMOS $0.25 \mu \mathrm{m} \mathrm{1P5M}$ \\
\hline Total Area & $34 \mathrm{~mm}^{2}, 25 \mathrm{~mm}^{2}$ (core) \\
RAM Area & $5.2 \mathrm{~mm}^{2}$ \\
\hline Maximum Speed & $50 \mathrm{MHz}$ \\
\hline Latency & $8221 @ N=8192$ \\
\hline Throughput & $1 \mathrm{sample} / \mathrm{cycle}$ \\
\hline Power Consumption & $300 \mathrm{~mW} @ 35 \mathrm{MHz}$ \\
\hline Package & CQFP144 \\
\hline Supply Voltage & $2.5 \mathrm{~V}$ \\
\hline
\end{tabular}

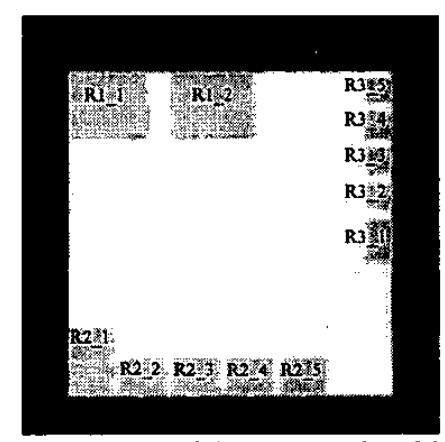

Fig. 5. Chip layout of the proposed architecture.

\section{CONCLUSION}

In this paper, the radix-4 DHT-based FFT algorithm is first derived according to the Hermitian symmetric property of the data in DMT-VDSL and ADSL systems, and the matrix form of the radix-2 DHT-based FFT designs [5][7]. Two PE circuits are proposed for variable-length application. One is composed of only one real multiplier and three adders for radix-2. The other is comprised with only one real multiplier and seven adders for radix-4. Finally, a variable-length $8192 / 4096 / 2048 /$ $1024 / 512$ radix-4 DIF DHT processor is demonstrated. It meets the DMT-VDSL and ADSL requirements and uses only six real multipliers, $2.01 N(16467)$ registers and some simple logic circuits. The chip is designed using $0.25 \mu \mathrm{m} 1 \mathrm{P} 5 \mathrm{M}$ typical CMOS technology and occupies a core area of $5 \mathrm{~mm} \times 5 \mathrm{~mm}$. Power dissipation is $300 \mathrm{~mW}$ under $2.5 \mathrm{~V}$ supply voltage while running at $35 \mathrm{MHz}$.

Table 3. Comparison with other FFT chips.

\begin{tabular}{|c|c|c|c|}
\hline & {$[9]$} & {$[10]$} & This Work \\
\hline FFT Size & 8192 & 8192 & $\begin{array}{c}8192 / 4096 / 2048 \\
/ 1024 / 512\end{array}$ \\
\hline Technology & $\begin{array}{c}0.5 \mu \mathrm{m} \\
\text { CMOS }\end{array}$ & $\begin{array}{c}0.6 \mu \mathrm{m} \\
\text { CMOS }\end{array}$ & $0.25 \mu \mathrm{m}$ CMOS \\
\hline $\begin{array}{c}\text { Chip Area } \\
\text { (core) }\end{array}$ & $1 \mathrm{~cm}^{2}$ & $140 \mathrm{~mm}^{2}$ & $34 \mathrm{~mm}^{2}$ \\
$107 \mathrm{~mm}^{2}$ & $25 \mathrm{~mm}^{2}$ \\
\hline $\begin{array}{c}\text { Normalized Area } \\
\text { (core) }\end{array}$ & $25 \mathrm{~mm}^{2}$ & $24.3 \mathrm{~mm}^{2}$ & $34 \mathrm{~mm}^{2}$ \\
\hline Clock & $20 \mathrm{MHz}$ & $20 \mathrm{MHz}$ & $35 \mathrm{~mm}^{2}$ \\
\hline $\begin{array}{c}\text { Power } \\
\text { Consumption }\end{array}$ & $600 \mathrm{~mW}$ & $650 \mathrm{~mW}$ & $300 \mathrm{~mW}$ \\
\hline $\begin{array}{c}\text { Word length: } \\
\text { Input/Output } \\
\text { sin, cos }\end{array}$ & $10 / 12$ & $10 / 12$ & $22 / 22$ \\
Internal results & 12 (bits) & 12 (bits) & 22 (bits) \\
\hline $\begin{array}{c}\text { Register } \\
\text { Delay- } \\
\text { line }\end{array}$ & RAM & SRAM \\
\hline \multicolumn{3}{|c|}{} & \\
\hline
\end{tabular}

\section{REFERENCES}

[1] D. J. Rauschmayer, ADSL/VDSL Principles, MTP, 1999.

[2] A. V. Oppenheim and R. W. Schafer, Discrete Time Signal Processing, Prentice Hall, 1999.

[3] S. He and M. Torkelson, "Design Pipeline FFT Processor for OFDM (de)Modulation," Int. Symp. on Signal, System, and Electronics, pp.257-262, 1998

[4] L. R. Rabiner and B. Gold, Theory and Application of Digital Signal Procession, Prentice Hall, Englewood Cliffs, New Jersey.

[5] C. L. Wang and C. H. Chang, "A DHT-based FFT/IFFT Processor for VDSL Transceivers," Int. Conf. on Acoustics, Speech and Signal Processing, Vol. 2, 2001.

[6] C. L. Wang, C. T. Ho and Y. T. Chang, "A novel systolic design for fast computation of the discrete Hartley transform," IEEE Thirtieth Asilomar Conf. on Signals, Systems \& Computers, pp. 1067-1071, Nov. 1996.

[7] C. L. Wang and C. H. Chang; "A novel DHT-based FFT/IFFT processor for ADSL transceivers," Int. Symp. on Circuits and Systems, Vol. 1, pp. 51 -54, 30 May, 1999.

[8] H. V. Sorensen, D. L. Jones, C. S. Burrus and M. T. Heideman, "On Computing the Discrete Hartley Transform", IEEE Trans. On Acoustic, Speech and Signal Processing, Vol. 33, pp. 1231-1238, Oct. 1985.

[9] E. Bidet, D. Castelain, C. Joanblanq, and P. Senn, "A Fast Single-Chip Implementation of 8192 Complex Point FFT", IEEE Journal of Solid-State Circuits, Vol. 30, No. 3, Mar. 1995

[10]L. Jia, "A New VLSI-Oriented FFT Algorithm and Implementation," IEEE ASIC Conference, pp. 337-341, 1998 